\title{
A 10-40 GHz 7 dB Directional Coupler in Digital CMOS Technology
}

\author{
Yunliang Zhu and Hui $\mathrm{Wu}^{*}$ \\ Laboratory for Advanced Integrated Circuits and Systems \\ Department of Electrical and Computer Engineering \\ University of Rochester, Rochester, NY 14627 \\ *Email: hwu@ece.rochester.edu
}

\begin{abstract}
We present an integrated wideband directional coupler for millimeter-wave applications implemented in lowcost silicon technologies. It is constructed using broad-side coupled coplanar waveguides (CPW), taking advantage of available multiple metal layers in silicon technologies. A prototype coupler was designed and fabricated using a commercial $0.18 \mu \mathrm{m}$ digital CMOS technology with low-resistivity epi substrate. It achieves a 10-40 $\mathrm{GHz}$ frequency range (3-dB bandwidth) with $7 \mathrm{~dB}$ to $10 \mathrm{~dB}$ coupling and more than $10 \mathrm{~dB}$ directivity. Measurement results match well with electromagnetic simulation.

Index Terms-CMOS, silicon, directional couplers, millimeter wave circuits, wideband
\end{abstract}

\section{INTRODUCTION}

There have been strong interests in using silicon technologies for integrated circuits at millimeter-wave frequencies [1][2][3][4]. A major reason is the low cost associated silicon technologies. More compelling is silicon's capability of integrating RF/microwave, analog, and digital circuits on a single chip, i.e., system-on-a-chip. It has become more feasible as recent progress in silicon technologies has pushed the cut-off frequency $\left(f_{T}\right)$ of NMOS transistors to over $150 \mathrm{GHz}$ at $90 \mathrm{~nm}$ technology node and beyond $200 \mathrm{GHz}$ at $65 \mathrm{~nm}$ [5]. However, wide adoption of silicon technologies at frequencies beyond $10 \mathrm{GHz}$ has been largely hindered by the lossy substrate, which results in large parasitic capacitance and high loss in onchip passive devices. This is particularly problematic in digital CMOS technologies, in which a low-resistivity substrate $(\sim 10 \mathrm{~m} \Omega \cdot \mathrm{cm})$ with a thin epitaxial layer is usually used to avoid latch-up and improve yield. Using bulk silicon substrate $(\sim 10 \Omega \cdot \mathrm{cm})$ alleviates the problem and is widely used for RF applications under $10 \mathrm{GHz}$. Further increasing the substrate resistivity poses problems in wafer yield and wafer cost. On the other hand, the progress in silicon technologies leads to increasing number of interconnect metal layers, and thicker top metals. This has opened up opportunities to reduce the loss in on-chip passive devices through innovative electromagnetic design.

Furthermore, recent advances in silicon-based millimeterwave ICs mainly focus on narrow-band applications [3][6]. There are even more challenges in the design and implementation of wideband MMICs using silicon technologies. One of them is the strong frequency dependency of on-chip passive device characteristics due to coupling to silicon substrate. For example, as frequency increases to millimeter-wave range, the spiral inductors may change the impedance from inductive to capacitive because of the self-resonance with parasitic

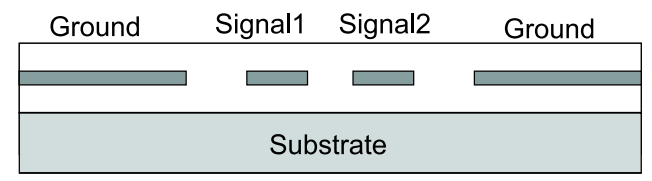

(a)

\begin{tabular}{|cll|}
\hline Ground & Signal1 & Ground \\
\hline & & \\
\hline Ground & Signal2 & Ground \\
\hline & Substrate & \\
\hline
\end{tabular}

(b)

Fig. 1. CPW directional couplers (not to scale). (a) edge-coupled; (b) broadside-coupled.

coupling capacitance to silicon substrate. In this work, we use a directional coupler as an example to investigate how to achieve large bandwidth for passive devices at millimeterwave frequencies using silicon technologies.

\section{Directional Coupler}

Directional couplers are important microwave passive devices for power division in various microwave circuits such as balanced amplifiers, balanced mixers, phase shifters, modulators, and measurement bridges. Branch-line couplers and hybrid ring couplers have been widely used as discrete devices or on printed circuit boards. Recently, a differential branch-line coupler has been implemented in a SiGe BiCMOS technology at $60 \mathrm{GHz}$ to generate quadrature local oscillator signals [6]. However, branch-line couplers and hybrids usually consume large chip area [7]. Therefore, coupled-line couplers are more suitable for MMIC implementations because of their smaller footprint.

The requirements for the transmission lines in a directional coupler are low loss and convenience to achieve different impedance levels. Microstrip lines and coplanar waveguides (CPWs) can both be implemented in silicon technologies. For microstrip lines, the signal line is usually built on the top metal layer, while the ground plane uses metal layers close to substrate. The achievable impedance level is usually low because of the small distance to the ground plane, and the minimum width limit for the signal line. CPWs can have both 
the signal and ground lines on the top metal layer, which usually is the thickest. The electromagnetic field is also moved further away from the lossy substrate. The impedance level of a CPW can be changed by adjusting the spacing between signal line and ground line. In addition, multiple metal layers can be stacked for the ground line to further reduce loss. Because of these reasons, coplanar waveguides are usually preferred for millimeter-wave circuits on silicon [1][8].

There are two major configurations for CPW directional couplers: edge-coupled [9], and broadside-coupled [10], as in Fig. 1. In silicon technologies, it is tempting to use the edge-coupled configuration since all transmission lines can then be constructed using the top metal layer, which is usually much thicker than lower metals. On the other hand, the gap between the two transmission lines in the edgecoupled configuration is limited by the design rule, which is significantly larger $(\sim 5-10 \mu m)$ for the thicker top metal layer. Hence, an interdigitated structure is usually needed in order to achieve enough coupling, which increases the chip area. In the broadside-coupled configuration, the gap between different metal layers is quite small $(\sim 1-2 \mu m)$ while the metal traces can be quite wide, which make strong coupling much easier to achieved. In addition, edge crowding effect increases the loss in the transmission lines, and largely offsets the benefits of using thicker metal for all transmission lines line. Thirdly, it is difficult for edge-coupled configuration to satisfy the metal density requirements mandated by chemical mechanical polishing (CMP) processes commonly used in silicon technologies because within the are of coupled transmission lines, only one metal layer is placed. Instead, broadside-coupled configuration can have stacked multiple metal layers which is easier to satisfy the metal density requirements. Therefore, broadside-coupled configuration is well suited for strong coupling in silicon technologies. In this paper, we present our work on a wideband broadside-coupled directional coupler using multiple interconnect metal layers in silicon technologies.

\section{DESIGN}

The cross section of the coupler is shown in Fig. 2-a, which shows the structure of the multi-layer CPW. Two signal lines are built on M6 and M4. In order to have a strong coupling, the M4 line is placed right under the M6 line in a symmetric fashion. To achieve other coupling coefficients, asymmetric structure can also be used. The design target for the coupling coefficient is $6 \mathrm{~dB}$, which implies the odd mode impedance is $28 \mathrm{Ohms}$ and the even mode impedance is 87 Ohms, given 50 Ohms system impedance. Coupled multi-layer CPW lines with different configurations are simulated in 3D EM simulator HFSS, which can account for the effect of metal layer thickness. The design parameters for the coupling coefficient and impedance levels are the width of upper signal line, the width of lower signal line and the spacing between signal line and ground, since the distance between two signal lines are fixed. The dimensions to achieve desired impedance levels are also shown in Fig. 2-a. The line length of the

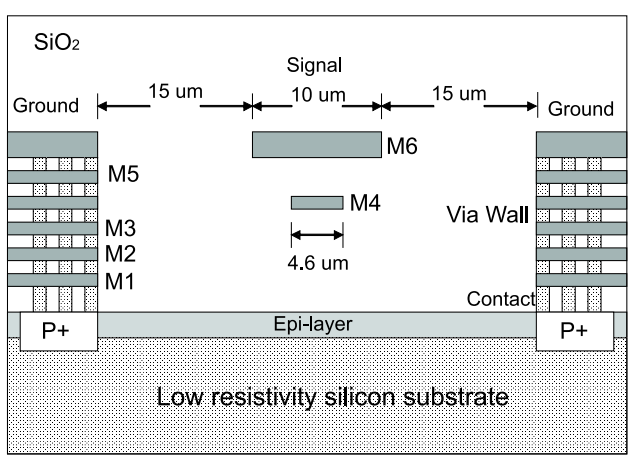

(a)

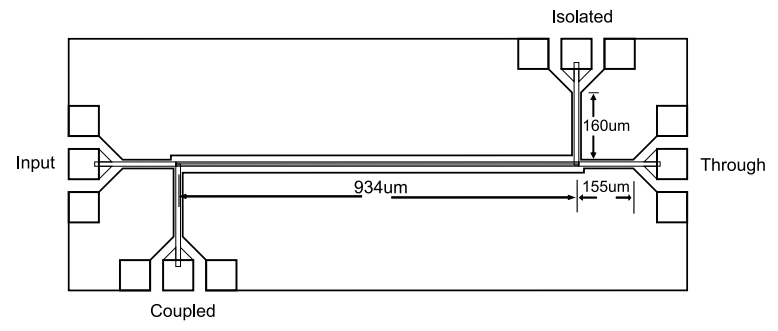

(b)

Fig. 2. Broadside-coupled CPW directional coupler in a digital CMOS process. (a) Cross section; (b) top view.

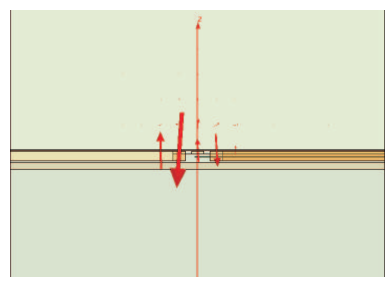

(a)

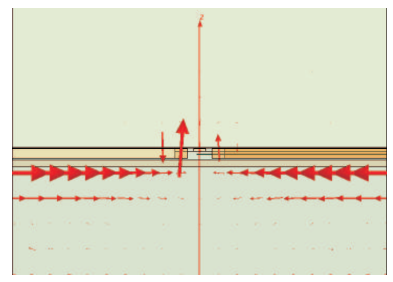

(c)

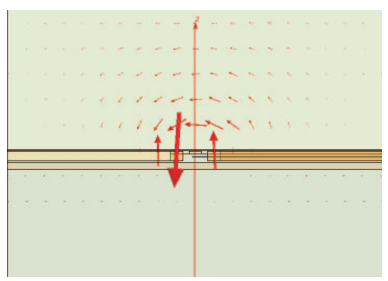

(b)

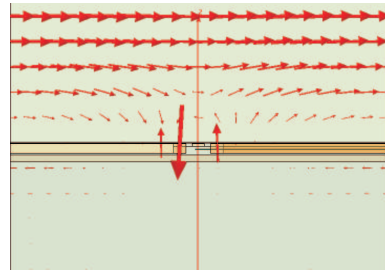

(d)
Fig. 3. Electric fields of possibly excited modes: (a) dominant quasi-TEM mode; (b) coupled slot-line mode; (c) parasitic substrate mode; (d) parasitic waveguide mode. 


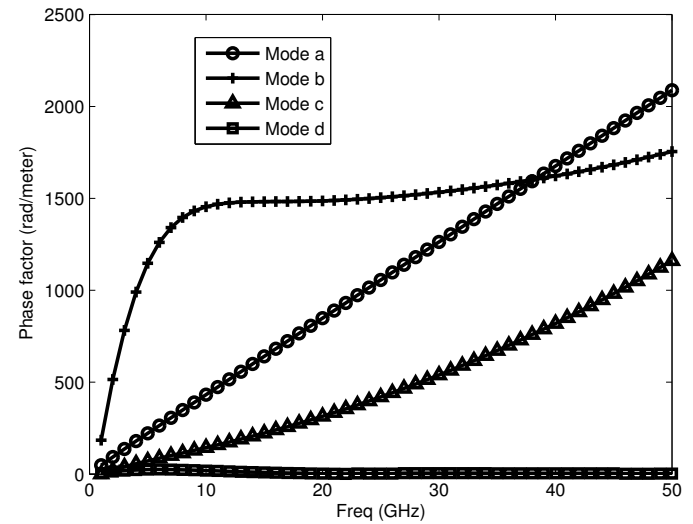

Fig. 4. Phase factors of possibly excited modes.

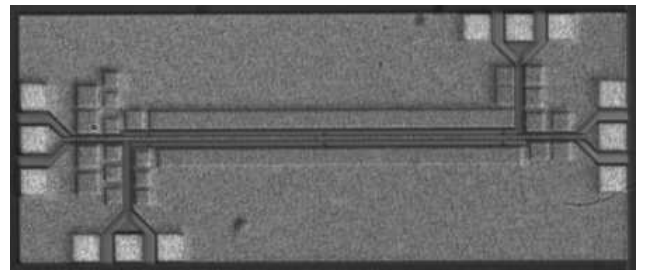

Fig. 5. Die micrograph of the coupler.

directional coupler is designed to be $934 \mu \mathrm{m}$ for a center frequency of $30 \mathrm{GHz}$. Because of the strong coupling between two signal lines, it is expected that this directional coupler would have a very wide bandwidth. The top view of the whole directional coupler is shown in Fig. 2-b.

From EM simulations, it is found that extra modes might be excited. Four modes which can be excited from the port, are shown in Fig. 3. The corresponding phase factors are shown in Fig. 4. From this phase factor plot, we can see that the first three modes are propagating and the fourth one is an evanescent mode. The first mode is the desired propagating mode for such a transmission line structure. The second mode is the coupled slot-line mode, which might occur in any CPW lines at the presence of any discontinuity. To suppress this coupled slot-line mode, under-pathes are connected between two ground lines to force them equal potential. The third one is a parasitic mode caused by the low resistivity silicon substrate. In order to suppress such a mode, many substrate contacts are added between ground lines and substrate to keep them equal potential. Two feed lines on M6 and M4 are also designed with the coupler with 50 Ohms impedance.

\section{Measurement Results}

The prototype coupler was fabricated in National Semiconductor's $0.18 \mu \mathrm{m}$ standard digital CMOS technology with low-resistance epi substrate. The chip photo is shown in Fig. 5. The chip size is $1.4 \mathrm{~mm}$ by $0.58 \mathrm{~mm}$. On the same die, two transmission lines with the same dimensions as feed lines which have $50 \mathrm{Ohms}$ impedance are also fabricated for

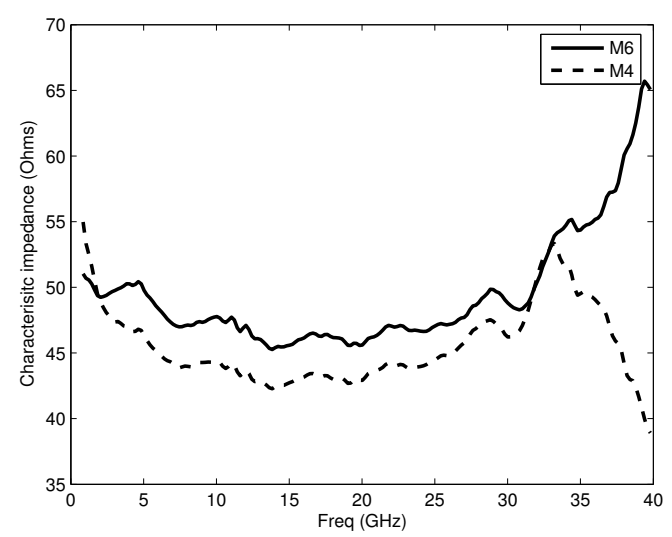

Fig. 6. Measured impedance levels of two feed lines.

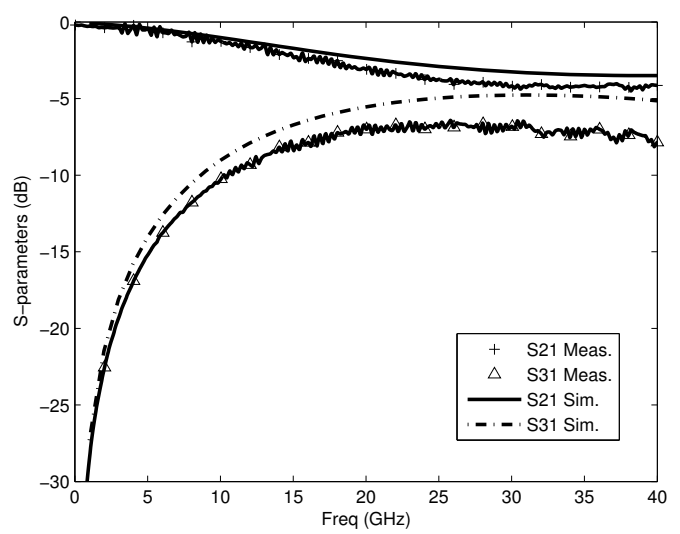

(a)

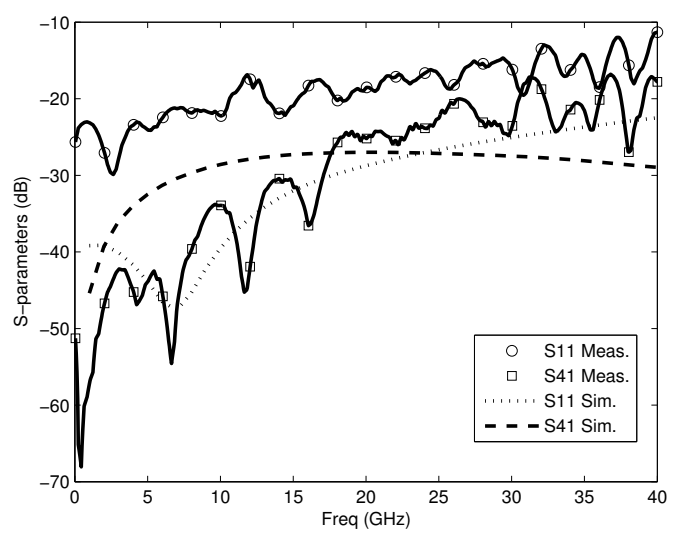

(b)

Fig. 7. Measured and simulated s-parameters of the coupler: (a) Through (S21) and Coupling (S31); (b) Matching (S11) and Isolation (S41). 


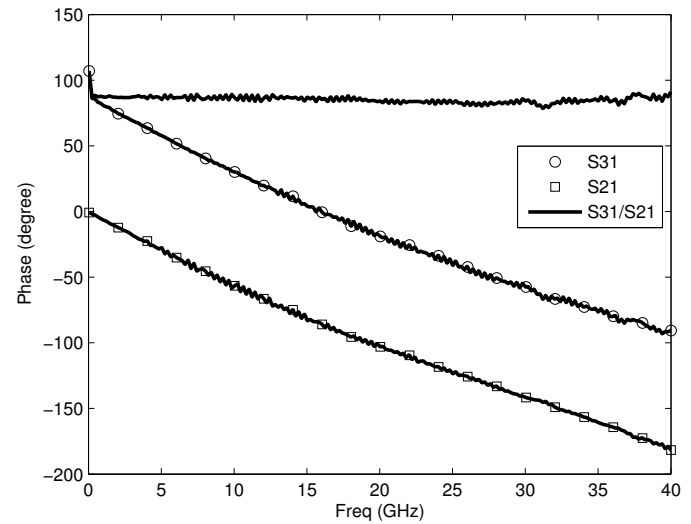

Fig. 8. Measured phase response of the coupler.

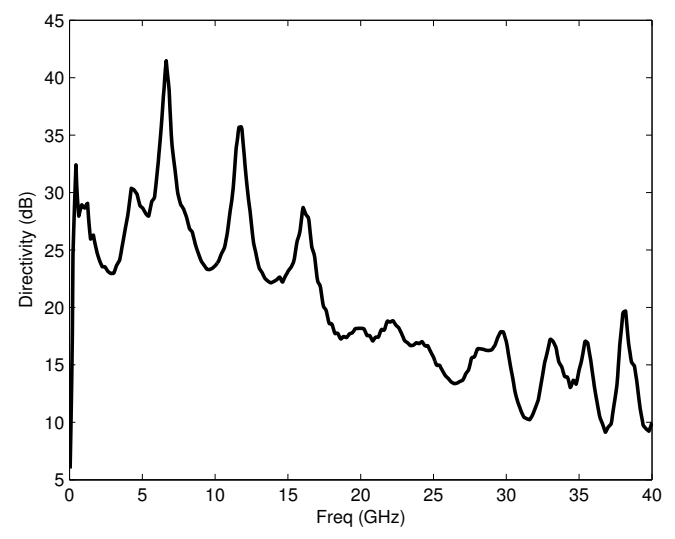

Fig. 9. Measured directivity of the coupler.

characterization purpose. One feed line is built on the top metal and the other is on Metal 4.

The measurement of the prototype coupler was performed in frequency domain up to $40 \mathrm{GHz}$ via on-wafer probing. The measured impedance levels of two feed lines are shown in Fig. 6. They match with design value $50 \mathrm{Ohms}$ well. This is very important for the coupler to have good terminations.

The measured and simulated s-parameters of the prototype coupler are both shown in Fig. 7 and Fig. 8 for both magnitude and phase response. The coupling coefficient is about $7 \mathrm{~dB}$ at $30 \mathrm{GHz}$, and it is flat within $20 \mathrm{GHz}$ to $35 \mathrm{GHz}$. The $-3 \mathrm{~dB}$ bandwidth can cover $10 \mathrm{GHz}$ to $40 \mathrm{GHz}$. The return loss $\mathrm{S} 11$ is lower than $-15 \mathrm{~dB}$ upto about $32 \mathrm{GHz}$ and stays below $11 \mathrm{~dB}$ up to $40 \mathrm{GHz}$. The isolation (S41) is more than $-20 \mathrm{~dB}$ to $30 \mathrm{GHz}$ and more than $-16 \mathrm{~dB}$ to $40 \mathrm{GHz}$. The phase response is quite linear, and the phase difference between through port (S21) and coupled port (S31) is quite close to 90 degrees within the whole bandwidth. As in Fig. 9, the directivity is $24 \mathrm{~dB}$ at $10 \mathrm{GHz}$, and is more than $13 \mathrm{~dB}$ up to $30 \mathrm{GHz}$. Until $40 \mathrm{GHz}$, the directivity can still be more than $10 \mathrm{~dB}$. The testing is performed up to $40 \mathrm{GHz}$; but as seen in Fig. 7.-a, the bandwidth is expected to extend beyond $40 \mathrm{GHz}$.

\section{CONCLUSiON}

We have designed and fabricated a wideband broad-side coupled CPW directional coupler using a commercial $0.18 \mu \mathrm{m}$ digital CMOS technology with low-resistivity epi substrate. The measurement results show $7 \mathrm{~dB}$ to $10 \mathrm{~dB}$ coupling and more than $10 \mathrm{~dB}$ directivity within $10-40 \mathrm{GHz}$. There are several ways to further improve the design. For example, the layout of broadside coupler can be meandered or spiraled to reduce the size [11]. To achieve larger directivity, shunt capacitors which are available in digital CMOS technology can be used to compensate phase velocity difference between even and odd modes. We can also customize the relative lateral position of two signal lines in an asymmetric configuration [12] to have different coupling coefficients. The proposed directional coupler can have a variety of applications in millimeter-wave integrated circuits for wireless communications, high-speed instrumentation, and radar systems.

\section{ACKNOWLEDGMENT}

The authors would like to thank Peter Holloway, Bijoy Chatterjee, Jun Wan, Babatunde Akinpelu, Peter Misich, Gabriele Manganreo, Sangamesh Buddhiraju, Jacque Margolycz and Gary Sheehan of National Semiconductor for their support in chip fabrication.

\section{REFERENCES}

[1] B. Kleveland, T.H. Lee, and S.S. Wong. $50 \mathrm{GHz}$ Interconnect Design in Standard Silicon Technology. In IEEE Microwave Symposium, pages 1913-1916, 1998.

[2] J. Kim, Y. Qian, G. Feng, P. Ma, M. F. Chang, and T. Itoh. MillimeterWave Silicon MMIC Interconnect and Coupler Using Multilayer Polyimide Technology. IEEE Trans. Microwave Theory Tech., 48(9):14821487, Sep. 2000.

[3] Hossein Hashemi, Xiang Guan, and Ali Hajimiri. A Fully Integrated $24 \mathrm{GHz} 8$-Path Phase-Array Receiver in Silicon. In IEEE Int. Solid-State Circuits Conf. Dig. Tech. Papers, pages 622-623, 2004.

[4] Talal Al-Attar, Arjang Hassibi, and Thomas H. Lee. A 77 GHz Monolithic IMPATT Transmitter in Standard CMOS Technology. In IEEE Microwave Symposium, pages 1423-1426, 2005.

[5] International Technology Roadmap for Semiconductors 2004 Update. http://public.itrs.net, 2004.

[6] S. Reynolds, B. Floyd, U. Pfeiffer, and T. Zwick. $60 \mathrm{GHz}$ Transceiver Circuits in SiGe Bipolar Technology. In IEEE Int. Solid-State Circuits Conf. Dig. Tech. Papers, pages 442-443, 2004.

[7] Khelifa Hettak, Gilbert A. Morin, and Malcolm G. Stubbs. Compact MMIC CPW and Asymmetric CPS Branch-Line Couplers and Wilkinson Dividers Using Shunt and Series Stub Loading. IEEE Trans. Microwave Theory Tech., 53(5):1624-1635, May 2005.

[8] H. Wu and A. Hajimiri. Silicon-based distributed voltage controlled oscillators. IEEE J. Solid-State Circuits, 36(3), Mar. 2001.

[9] C.P. Wen. Coplanar-Waveguide Directional Couplers. IEEE Trans. Microwave Theory Tech., 18(6):318-322, Jun. 1970.

[10] C. Nguyen. Broadside-Coupled Coplanar Waveguides and Their EndCoupled Band-Pass Filter Applications. IEEE Trans. Microwave Theory Tech., 40(12):2181-2189, Dec. 1992.

[11] Shih-Ming Wang, Chin-Hsiung Chen, and Chi-Yang Chang. A Study of Meandered Microstrip Coupler with High Directivity. In IEEE Microwave Symposium, pages 63-66, 2003.

[12] C. Nguyen. Analysis of Unsymmetrical Broadside-coupled Coplanar Waveguides for Microwave Integrated Circuits. In Digest of Antennas and Propagation Society International Symposium, pages 2222-2224, 1993. 